\title{
An engineering model and its numerical validation for a malevolent aircraft impinging against a rigid target: Force and impulse estimations
}

\author{
Tieping $\mathrm{Li}^{\mathrm{a}, 1}$, Wei Wang ${ }^{\mathrm{b}, \mathrm{c}, 1}$, Fuping Yuan ${ }^{\mathrm{b}, \mathrm{c}, *}$, Xiaolei $\mathrm{Wu}^{\mathrm{b}, \mathrm{c}}$ \\ ${ }^{a}$ Nuclear and Radiation Safety Center, Ministry of Environmental Protection of the People's Republic of China, No. 54 Hongliannancun, Haidian District, Beijing 100082, \\ People's Republic of China \\ ${ }^{\mathrm{b}}$ State Key Laboratory of Nonlinear Mechanics, Institute of Mechanics, Chinese Academy of Sciences, No. 15, North 4th Ring, West Road, Beijing 100190, People's \\ Republic of China \\ ${ }^{\mathrm{c}}$ School of Engineering Science, University of Chinese Academy of Sciences, Beijing 100190, People's Republic of China
}

G R A P H I C A L A B S T R A C T
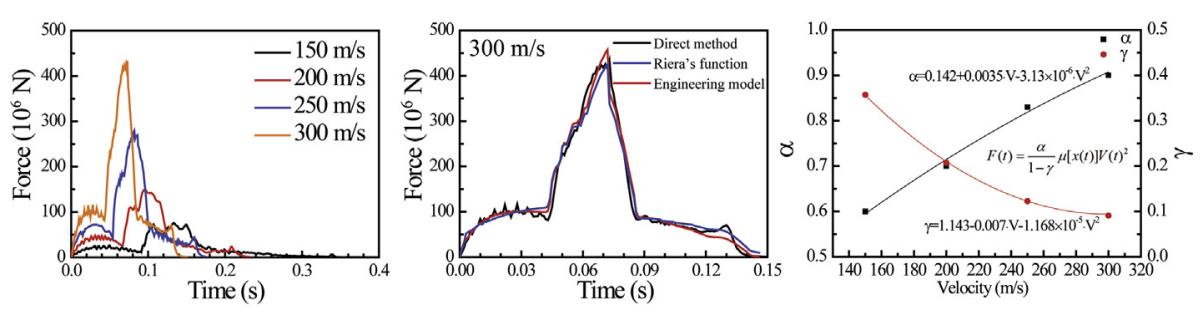

\section{A R T I C L E I N F O}

\section{Keywords:}

Aircraft impact

Numerical simulation

Impact force

Riera's model

Finite element method

Crushing force

\begin{abstract}
A B S T R A C T
Generally, the time-dependent impact force of a commercial aircraft against a rigid wall can be calculated based on the Riera's model, which consists of the static crushing force and the dynamic force. The reduction coefficient $\alpha$ is generally considered to be less than a unity in the modified Riera's function, while the influence of the impact velocity on the reduction coefficient is not investigated before. Moreover, the static crushing force is hard to be determined in practice. Thus an engineering model was proposed in the present study, and the total force was only described by the dynamic force and another velocity-dependent coefficient $\gamma$. Based on a well-defined finite element model for the commercial aircraft Boeing B737-800, the entire impact process of the aircraft impinging on a rigid wall was reproduced by the commercial software LS-DYNA, and four different impact velocities $(150,200,250,300 \mathrm{~m} / \mathrm{s})$ were considered. Based on the simulation results, the influences of impact velocity on the two coefficients ( $\alpha$ and $\gamma$ ) were investigated and discussed by matching the aircraft impact force/ impulse with the reaction force/impulse of the rigid wall. Finally, two formulas for the effects of impact velocity on the two coefficients were proposed, which is helpful for the engineers in designing and assessing the protecting shell of the nuclear power plant.
\end{abstract}

\section{Introduction}

In the last several decades, the crashworthiness design and the safety assessment for the protecting shell of nuclear power plants (NPP) subjected to the malevolent aircraft impact have attracted great interests due to the increasing threats of the terrorist attacks and the rapidly

* Corresponding author at: State Key Laboratory of Nonlinear Mechanics, Institute of Mechanics, Chinese Academy of Sciences, No. 15, North 4th Ring, West Road, Beijing 100190, People's Republic of China.

E-mail address: fpyuan@lnm.imech.ac.cn (F. Yuan).

${ }^{1}$ T.P. Li and W. Wang contributed equally to this work. 
growing numbers of NPP (Abbas et al., 1996; Itoh et al., 2005; Li et al., 2005; Arros and Doumbalski, 2007; Petrangeli, 2010; Frano and Forasassi, 2011; Iqbal et al., 2012; Jeon et al., 2012; Lee et al., 2013; Sadique et al., 2013; Kostov et al., 2014; Siefert and Henkel, 2014; Tennant et al., 2014; Andonov, et al., 2015; Lu et al., 2015; Thai and Kim, 2015; Jeon and Jin, 2016; Hu et al., 2017; Yu et al., 2017; Zhang et al., 2017; Hu et al., 2018; Wang et al., 2018). The histories of the impact force and the impact impulse have to be estimated from the expected impact velocities in order to assess the effects of aircraft impacts on the protecting shell of NPP.

Based on a rigid-perfectly plastic materials model, Riera (1968) proposed the first one-dimensional theoretical formula to obtain the time history of the impact force by considering normal cylinder impact upon a rigid wall. Since then, Riera's function has been widely used, and several researchers have also tried to modify the Riera's model for improving the accuracy of impact force calculation (Dritter and Gruner, 1976; Hornyik, 1977; Bahar and Rice, 1978; Wolf et al., 1978; Kar, 1979; Riera, 1980; Riera, 1982; Rambach et al., 2005). For example, a slightly different method for calculating the impact force have been proposed by Dritter and Gruner (1976), in which the rigid-perfectly plastic materials model has been replaced by an elastic-plastic materials model for the aircraft. While, Wolf et al. (1978) has obtained the similar results with those by the Riera's function by proposing a lumped massspring model. By considering the losses of energy and mass from the broken flying debris, more modifications to the Riera's model (Hornyik, 1977; Bahar and Rice, 1978; Kar, 1979; Riera, 1980; Riera, 1982; Sugano et al., 1993; Rambach et al., 2005) have been proposed. These studies have indicated that the reduction coefficient $\alpha$ in the Riera's function should be less than a unity to capture accurately the aircraft impact force. However, this reduction coefficient should be highly related to the degree of flying debris and the impact velocity, thus the relationship between the reduction coefficient and the impact velocity should be further investigated. Moreover, the impulse of the dynamic force in the total pulse is the easy part to be obtained, while the impulse of the crushing force is hard to be determined for engineering applications. Thus, an engineering model is needed in which the total pulse can only be described by the impulse of the dynamic force, once the coefficient $\gamma$ between the impulse of the crushing force and the total impulse is determined through a series of numerical simulations.

No open prototype impact test using large commercial aircrafts has been reported so far due to the complex experimental techniques and the expensive costs. While, finite element method (FEM) has been successfully implemented to obtain the time history data of aircraft impact by building high-quality FE aircraft models (Abbas et al., 1996; Petrangeli, 2010; Frano and Forasassi, 2011; Iqbal et al., 2012; Lee et al., 2013; Sadique et al., 2013; Kostov et al., 2014; Lu et al., 2015; Thai and Kim, 2015; Jeon and Jin, 2016). The previous studies (Itoh et al., 2005; Siefert and Henkel, 2014; Lu et al., 2015) have also indicated that the geometrical structure of the aircraft in FE models should be close to the actual structures as much as possible in order to accurately obtain the time history of impact force. In this paper, highquality FE models for the Boeing B737-800 commercial aircraft impinging on a rigid wall were established, and then a series of numerical simulations based on the missile-target interaction analysis (Lee et al., 2013) have been conducted to obtain the loading-time history data. Then, an engineering model was established, and impact velocity effects on the corresponding coefficients of $\alpha$ and $\gamma$ have been investigated.

\section{FE models}

In this section, FE models for the Boeing B737-800 commercial aircraft impinging on a rigid target have been established using the mesh generation software HyperMesh (Altair HyperWorks, 2010) and the commercial FE software LS-DYNA (Hallquist et al., 2013).

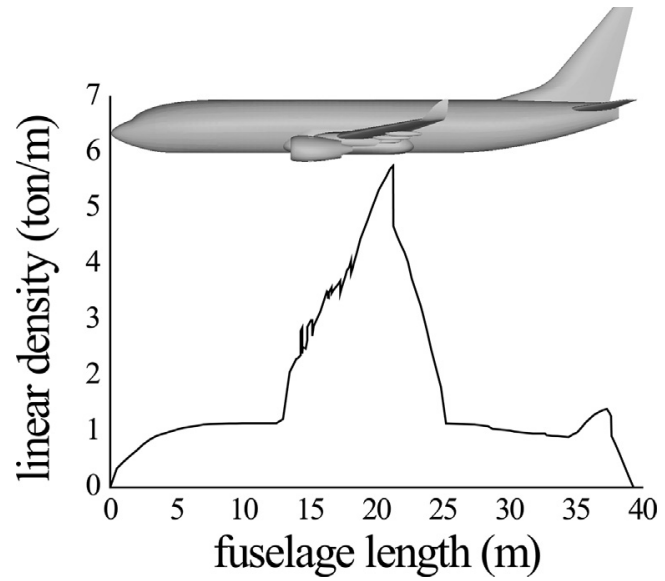

Fig. 1. Mass distribution along the fuselage length for the built Boeing B737800 commercial aircraft model.

\subsection{Boeing B737-800 commercial aircraft}

Boeing B737-800 aircraft is a widely used in-service commercial aircraft all over the world, and it has a length of fuselage of $39.5 \mathrm{~m}$, a width of wingspan of $38.5 \mathrm{~m}$ and a height of $12.5 \mathrm{~m}$ (http:// www.boeing.com). The weight of Boeing B737-800 aircraft is about 70.533 tons (Song and Li, 2016). The geometrical structures of FE models are nearly impossible to be totally identical to the actual structures of aircraft due to the complexity of the internal structure. Thus, reasonable simplification in FE models should be considered since the impact force is mainly related to the distributions of the mass and the stiffness based on the Riera's model. Fig. 1 displays the mass distribution (linear density, ton/m) along the fuselage length for the built aircraft model, and the contour of the aircraft is also shown in the figure. This mass distribution has good agreement with the open data from the Boeing company and has similar shape with the previous papers for other commercial aircrafts (Kostov et al., 2014; Thai and Kim, 2015; Zhang et al., 2017). In the model, the two wings (including aviation fuel) have a weight of 24.40 tons, the two horizontal tails have a weight of 1.974 tons, the vertical tail has a weight of 2 tons, the two engines have a weight of 4.726 tons, and the outer skin has a weight of 37.433 tons. It is observed from the figure that the position for the maximum linear density is coincided with the position of the power system (wings and engines). Based on the distribution of the stiffness, the main components of aircraft were considered as follows: (i) Fuselage stringers, fuselage frames and floor beams; (ii) Ribs, beams and stringers for the two wings, the two horizontal tails and the vertical tail; (iii) Envelope for entire aircraft; (iv) Two engines. Except the aircraft envelope, the geometrical structures of FE models, are shown in Fig. 2.

After building geometry for the aircraft, mesh generation was realized using software HyperMesh (Altair HyperWorks, 2010). The illustration for the mesh generation is displayed in Fig. 3a. The aircraft consists of shell elements for the shell components (envelope, engine) and beam elements for the other components. The Belytschko-Tsay algorithm was used in shell elements, and single point integral was utilized by hourglass control. While, the cross section integral was utilized in beam elements. The rigid wall has a length of $60 \mathrm{~m}$, a width of $60 \mathrm{~m}$, and a thickness of $1 \mathrm{~m}$. The element size is $1 \mathrm{~m}$ in the rigid wall. In the aircraft, the element size for the main body is $250 \mathrm{~mm}$, the element size for the engine is $50 \mathrm{~mm}$.

\subsection{Materials models}

The materials for the main structures in Boeing B737-800 aircraft are steel alloy (engine) and aluminum alloy (main body). According to the previous paper (Lu et al., 2015), the elastic constants for both steel 


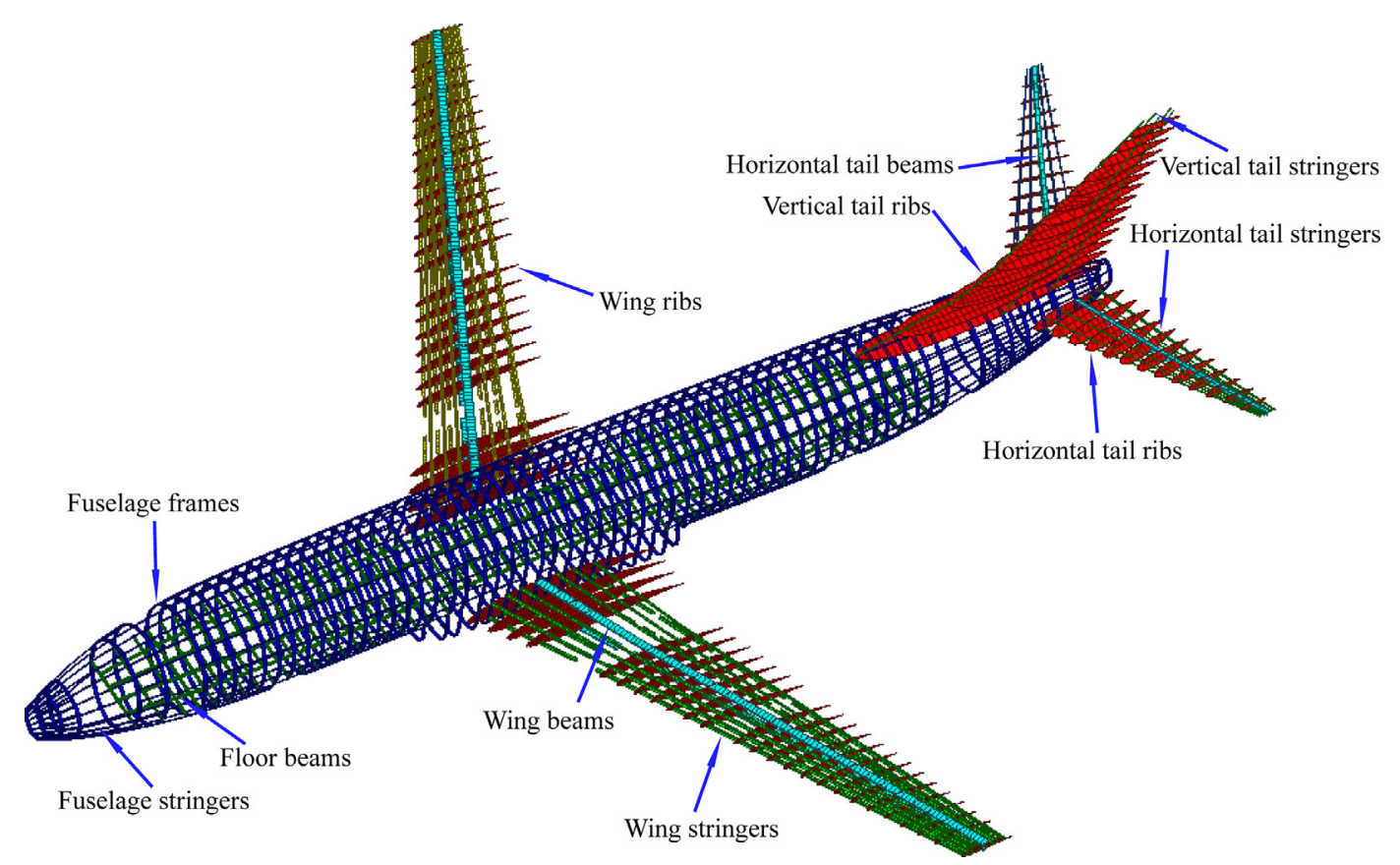

Fig. 2. Geometrical structures of FE models except the aircraft envelope for the Boeing B737-800 commercial aircraft.

alloy and aluminum alloy are listed in Table 1. In high-speed impact, both strain rate and thermal effects on the materials can't be ignored and should be considered, thus both Johnson-Cook model and plastickinematic model were used in the present study. Johnson-Cook model was used for all shell elements (envelope with aluminum and engine with steel), while plastic-kinematic model was used for all beam elements (other components).

A computational model, known as Johnson-Cook model, is presented here for describing all effects of strain hardening, strain rate hardening and thermal softening on the flow stress. This model is quite useful due to its simple expression for engineering applications, and the von Mises flow stress can be expressed as three uncoupled terms as following:

$\sigma=\left[A+B \varepsilon_{p}^{n}\right]\left[1+C \ln \dot{\varepsilon}^{*}\right]\left[1-T^{* m}\right]$

where $\varepsilon_{p}$ is the true plastic strain, $\dot{\varepsilon}^{*}=\dot{\varepsilon} / \dot{\varepsilon}_{0}$ is the normalized strain rate with respect to $\dot{\varepsilon}_{0}=1 / \mathrm{s}$, and $T^{*}$ is the homologous temperature defined as $T^{*}=\left(T-T_{\text {room }}\right) /\left(T_{\text {melt }}-T_{\text {room }}\right) . A, B, C, n, m$ are five material parameters, which need to be determined by fitting the experimental data.

The failure in the Johnson-Cook model is determined by $D=1$, and $D$ can be expressed as following:

$D=\sum_{i=1}^{N} \frac{\Delta \varepsilon_{i}}{\left(\varepsilon_{f}\right)_{i}}$

where $\Delta \varepsilon_{i}$ is the equivalent plastic strain increment in the $\mathrm{i}_{\text {th }}$ time step, $\left(\varepsilon_{f}\right)_{i}$ is the failure strain in the $\mathrm{i}_{\text {th }}$ time step and $N$ is the total step number. $\varepsilon_{f}$ can be expressed as following:

$\varepsilon_{f}=\left[D_{1}+D_{2} \exp \left(D_{3} \sigma^{*}\right)\right]\left[1+D_{4} \ln \dot{\varepsilon}^{*}\right]\left[1+D_{5} T^{*}\right]$

where $\sigma^{*}=\frac{\sigma_{m}}{\bar{\sigma}}\left(\sigma_{m}\right.$ is the mean stress and $\bar{\sigma}$ is the von Mises flow stress), $D_{1} \sim D_{5}$ are five material parameters, which need to be determined by experimental data. According to the previous paper (Lu et al., 2015), the materials constants for the Johnson-Cook model of steel (engine) and aluminum (envelope) alloys are listed in Table 2. Thus, the failure of the element is realized by $D=\sum_{i=1}^{N} \frac{\Delta \varepsilon_{i}}{\left(\varepsilon_{f}\right)_{i}}=1$.

Plastic-kinematic model is also a model considering all effects of strain hardening, strain rate hardening and thermal softening on the flow stress, and it can be expressed as following:
$\sigma=\left[1+\left(\frac{\dot{\varepsilon}}{C p}\right)\right]\left(\sigma_{0}+\beta E_{p} \varepsilon_{p}^{e f f}\right)$

where $\sigma_{0}$ is the yield stress, $\varepsilon_{p}^{e f f}$ is the equivalent plastic strain, $\dot{\varepsilon}$ is the strain rate, $E_{p}$ is the plastic strain hardening modulus $\left(E_{p}=\frac{E E_{t}}{E-E_{t}}\right.$, where $E_{t}$ is the tangent modulus), and $C, p$ are materials parameters. The parameter $\beta=0$ represents kinematic hardening, $\beta=1$ represents isotropic hardening and $\beta$ with other value represents mixed hardening. The elements fail when the equivalent plastic strain reaches the parameter $F_{s}$. According to the previous paper (Lu et al., 2015), the materials constants for the plastic-kinematic model of aluminum alloy (other components) are listed in Table 3. As indicated, the aluminum used for the envelope (Johnson-Cook model) has a lower yield strength when compared to the aluminum used for the other components (Plastic-kinematic model).

\subsection{Contacts}

There exist three contact types in the simulations: (i) The keyword "CONTACT_AUTOMATIC_BEAMS_TO_SURFACE" is used to define the contact between the beam structures and the rigid wall; (ii) The keyword "CONTACT_AUTOMATIC_SURFACE_TO_SURFACE" is utilized to define the contact between the shell structures and the rigid wall; (iii) The self contact for the aircraft itself after large plastic strain is realized by the keyword "CONTACT_AUTOMATIC_SINGLE_SURFACE". The coefficient of friction of 0.1 is used in all three contact types.

\subsection{Verification for element size}

When the mesh size is too small, the computational time is too long. While, the results are not accurate when the mesh size is too large. Thus, convergence of the computations was checked by comparing the present results (with impact velocity of $300 \mathrm{~m} / \mathrm{s}$ ) with those calculated using a finer mesh (half size). Illustrations of the mesh generation in the fuselage and the engine for the present FE model are shown in Fig. 3b and $\mathrm{d}$, while illustrations of the mesh generation in the fuselage and the engine for the FE model with a finer mesh are displayed in Fig. 3c and e. The mesh sizes of fuselage are $250 \mathrm{~mm}$ and $125 \mathrm{~mm}$ in Fig. 3b and c, while the mesh sizes of engine are $50 \mathrm{~mm}$ and $25 \mathrm{~mm}$ in Fig. 3d and e. 


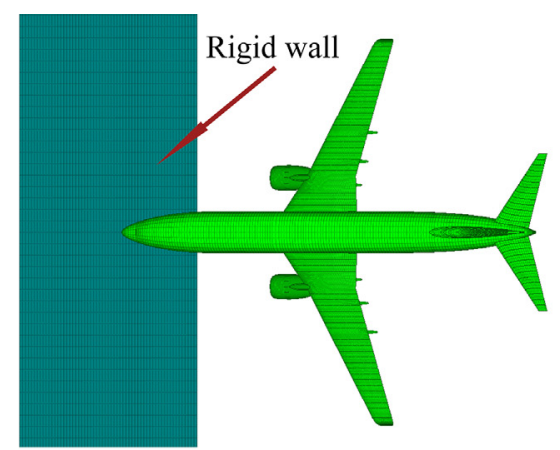

(a)

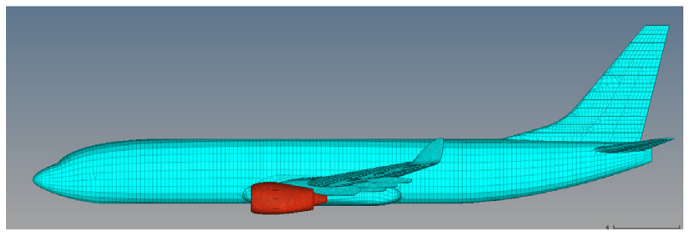

(b)

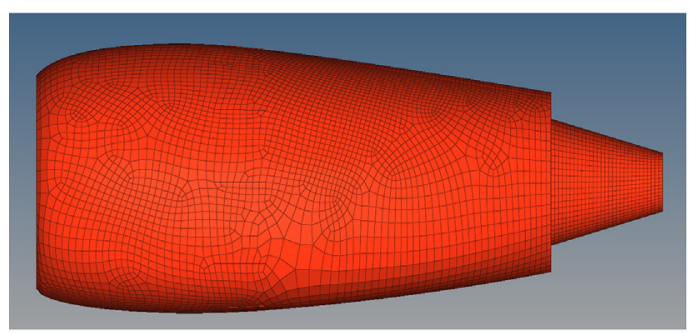

(d)

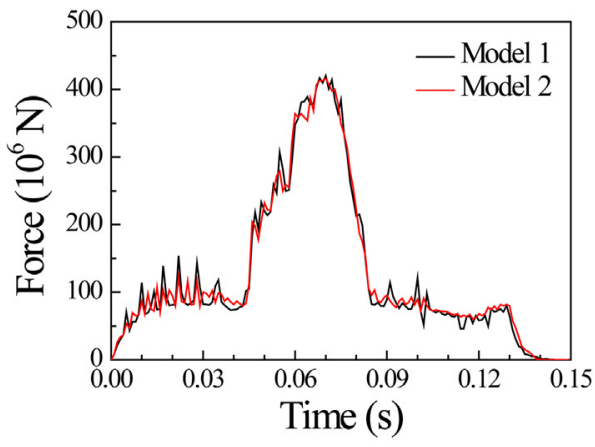

(f)

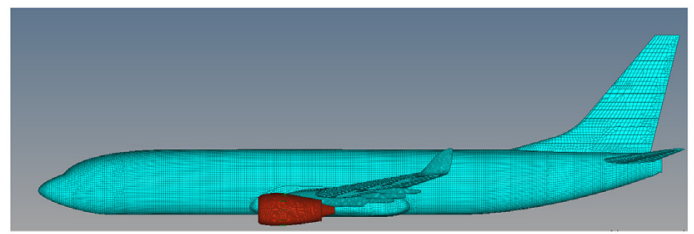

(c)

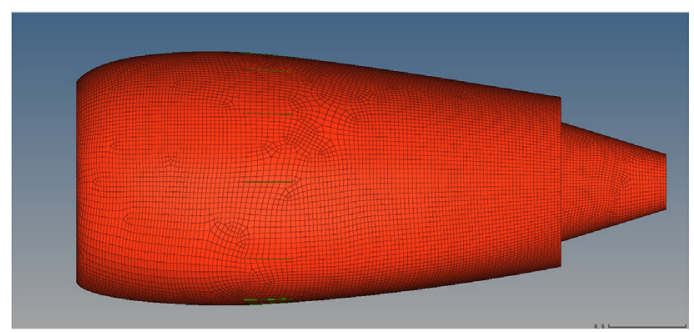

(e)

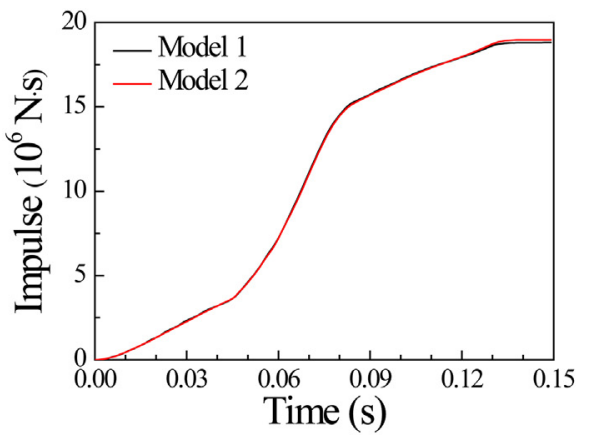

(g)

Fig. 3. (a) Illustration of the mesh generation for the present FE model. (b) Illustration of the mesh size in the fuselage for the present FE model. (c) Illustration of the mesh size in the fuselage for the FE model with a finer mesh. (d) Illustration of the mesh size in the engine for the present FE model. (e) Illustration of the mesh size in the engine for the FE model with a finer mesh. Verification for the convergence of the computations by comparing the present results (at impact velocity of $300 \mathrm{~m} / \mathrm{s}$ ) with those calculated using a finer mesh (half size): (f) Time histories of impact force; ( $g$ ) Time histories of impulse.

Table 1

Elastic constants for steel and aluminum alloys.

\begin{tabular}{lll}
\hline & Steel & Aluminum \\
\hline Density $\left(\mathrm{kg} / \mathrm{m}^{3}\right)$ & 7800 & 2800 \\
Young's modulus (GPa) & 210 & 71.9 \\
Poisson's ratio & 0.3 & 0.33 \\
\hline
\end{tabular}

The time histories of force and impulse for the current mesh size (model 1) and the finer mesh size (model 2) are displayed in Fig. 3f and g. As indicated, the two mesh sizes have virtually given the same curves (the force curves have only an error of $2.4 \%$ and the impulse curves are almost identical), indicating that the current mesh size is fine enough.

\section{Review of Riera's model and proposal of engineering model}

In Riera's model, the impact force exerted on the rigid target, $F(t)$, can be expressed as following, when a cylinder impacts a rigid target:

$F(t)=P_{c}[x(t)]+\mu[x(t)] V(t)^{2}$

where the first term of the right side $\left(P_{c}[x(t)]\right)$ is the crushing force (contribution from the static pressure), and the second term of the right side is the dynamic force (contribution from the dynamic pressure). $\mu[x(t)]$ is the mass per unit length and $V(t)$ is the instantaneous 
Table 2

Materials parameters for Johnson-Cook model.

\begin{tabular}{lll}
\hline & Steel & Aluminum \\
\hline A (MPa) & 350 & 369 \\
B (MPa) & 275 & 684 \\
$\mathrm{C}$ & 0.022 & 0.0083 \\
$\mathrm{~m}$ & 1.0 & 1.7 \\
$\mathrm{n}$ & 0.36 & 0.73 \\
$\mathrm{D}_{1}$ & 0.05 & 0.13 \\
$\mathrm{D}_{2}$ & 3.44 & 0.13 \\
$\mathrm{D}_{3}$ & -2.12 & -1.5 \\
$\mathrm{D}_{4}$ & 0.002 & 0.011 \\
$\mathrm{D}_{5}$ & 0.61 & 0 \\
\hline
\end{tabular}

Table 3

Materials parameters for plastic-kinematic model.

\begin{tabular}{ll}
\hline & Aluminum \\
\hline$\sigma_{0}(\mathrm{MPa})$ & 503 \\
$E_{t}(\mathrm{MPa})$ & 5000 \\
$\beta$ & 0 \\
$C(1 / \mathrm{s})$ & 40 \\
$p$ & 5 \\
$F_{S}$ & 0.05 \\
\hline
\end{tabular}

crushing velocity of the aircraft. Generally, the contribution of the crushing load to the total force is not significant.

Generally, the actual impact force should be less than the right side of Eq. (5) due to the energy loss. Thus, a reduction coefficient $\alpha$ was introduced (Kar, 1979) and the Eq. (5) can be rewritten as following:

$F(t)=P_{c}[x(t)]+\alpha \mu[x(t)] V(t)^{2}$

The Riera's model is an indirect method using the load function, while the so-called "missile-target interaction analysis" (Lee et al., 2013) utilizes the verified FE simulations to acquire the reaction force of the rigid wall directly. In previous paper, the reduction coefficient $\alpha$ was empirically determined to be 0.9 (Sugano et al., 1993). However, the degree of flying debris and the impact velocity should have great influences on this reduction coefficient, therefore the relationship between the reduction coefficient and the impact velocity is investigated in the present study.

In practice, the dynamic force in the total force is the relatively easy part to be determined, while the static crushing force is a little harder to be obtained. Therefore, we propose an engineering model for easy use of load function in practice. The time duration of the crushing force and the impact force is the same, and the crushing load generally does not affect the magnitude of the total impact force significantly, thus the ratio between the instantaneous static impulse $\left(I_{P}\right)$ and the instantaneous total impulse $\left(I_{F}\right)$ can be assumed to be a constant and defined as a coefficient $\gamma$. Thus, Eq. (6) can be rewritten as:

$F(t)=\frac{\alpha}{1-\gamma} \mu[x(t)] V(t)^{2}$

The engineering model avoids the difficulty for obtaining the static crushing force, and the total force is only described by the dynamic force. The two coefficients can be determined through a series of numerical simulations by matching both the force and the impulse histories for both the direct method and the indirect methods (the modified Riera's model and the engineering model).

\section{Results and discussions}

The studies of aircraft impact events with different impact velocities have been considered in previous research (Dritter and Gruner, 1976; Zorn and Schueller, 1986; Sugano et al., 1993; Abbas et al., 1996; Arros
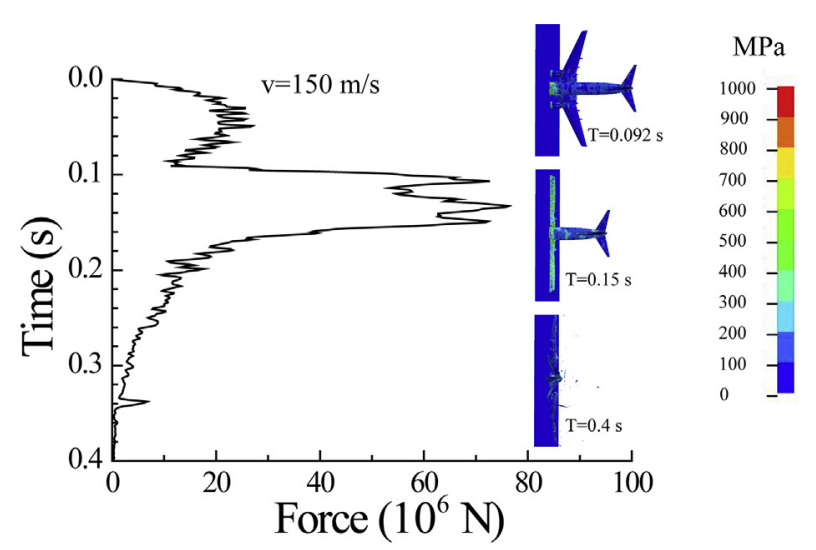

(a)

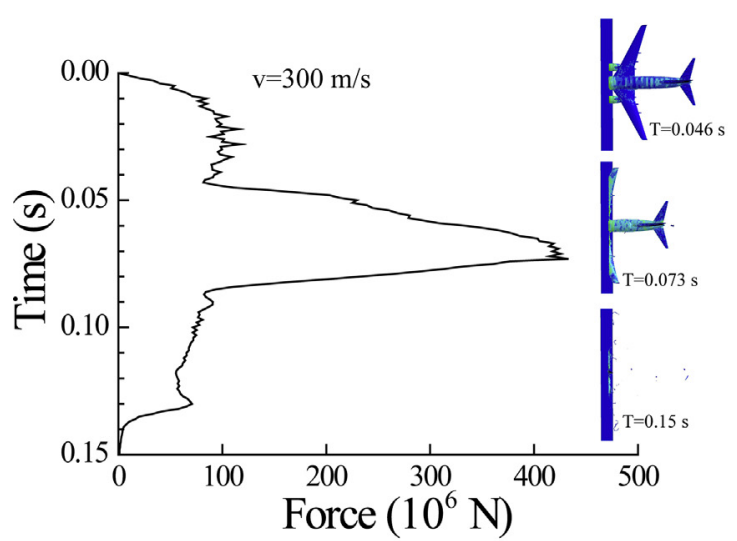

(b)

Fig. 4. Time histories of impact force for the simulations along with the stress contours for the whole craft at three typical times, at two different impact velocities: (a) $150 \mathrm{~m} / \mathrm{s}$; (b) $300 \mathrm{~m} / \mathrm{s}$.

and Doumbalski, 2007; Petrangeli, 2010; Frano and Forasassi, 2011; Wilt et al., 2011; Iqbal et al., 2012; Jeon et al., 2012; Lu et al., 2015; Thai and Kim, 2015; Jeon and Jin, 2016). The impact velocities are 235, 247, $293 \mathrm{~m} / \mathrm{s}$ in the September 11 event, while the most previously considered impact velocities were less than $220 \mathrm{~m} / \mathrm{s}$. In normal landing and takeoff events, there exists a speed limit for commercial aircrafts, while an intentional aircraft crashing into NPP might have higher impact speed. Thus, based on the aforementioned FE models, the entire impact process of an aircraft impacting on a rigid wall is reproduced by the LS-DYNA, and four different impact velocities (150, $200,250,300 \mathrm{~m} / \mathrm{s}$ ) are utilized. The effects of impact velocity on the impact force and the coefficients ( $\alpha$ and $\gamma$ ) will be discussed.

Fig. 4 shows the time histories of force for the simulations at two different impact velocities ( 150 and $300 \mathrm{~m} / \mathrm{s}$ ), along with the stress contours for the whole craft at three typical times. The impact force was obtained by the direct method (i.e., the reaction force of the rigid wall). As we know, the impact force is induced by three main components of the aircraft (fuselage, two wings and two engines). As shown in Fig. 4, the impact force induced by fuselage has a relatively lower value, sustaining the entire crushing process. The peak force occurs as the two engines and two wings impact on the rigid wall, and the durations for the impacts of engines and wings are about 0.1 and $0.005 \mathrm{~s}$ for the simulations with impact velocities of $150 \mathrm{~m} / \mathrm{s}$ and $300 \mathrm{~m} / \mathrm{s}$, respectively. Total durations for the impact of the entire aircraft are about 0.35 and $0.15 \mathrm{~s}$ for the simulations with impact velocities of $150 \mathrm{~m} / \mathrm{s}$ and $300 \mathrm{~m} / \mathrm{s}$, respectively. As mentioned earlier, energy loss and mass loss should be induced by the crushing of the airframe and the erosion effect of the aircraft debris, thus the coefficient $\alpha$ should be less than a unity. Only the area close to the impact contact surface has the plastic 


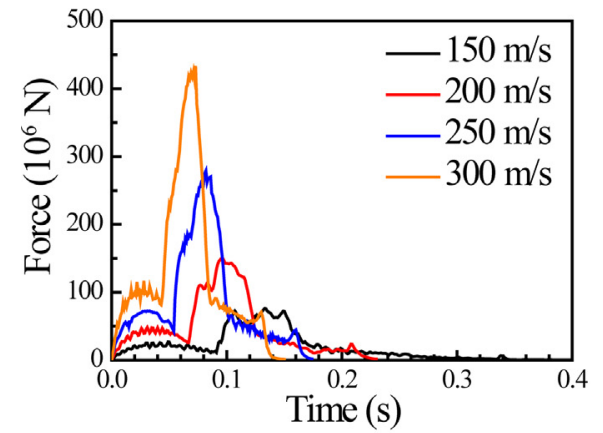

(a)

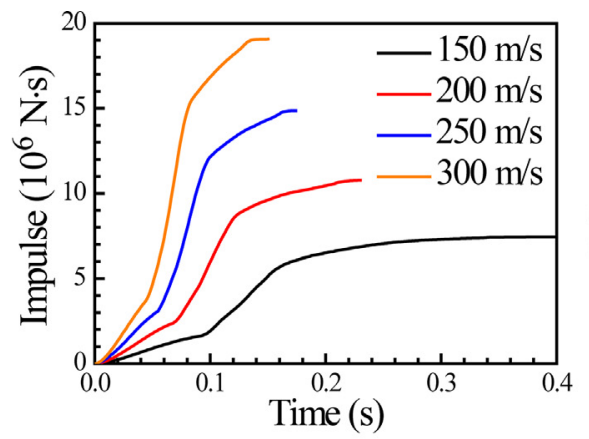

(b)

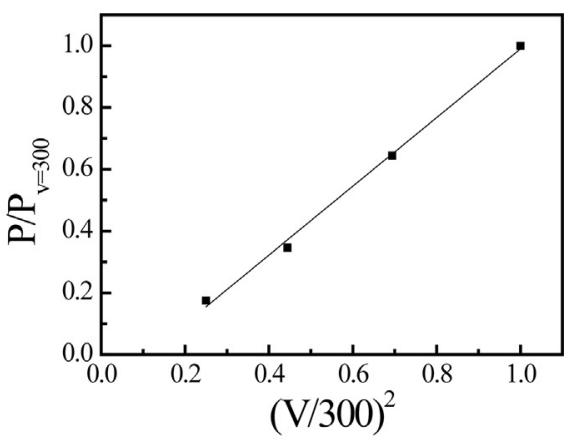

(c)

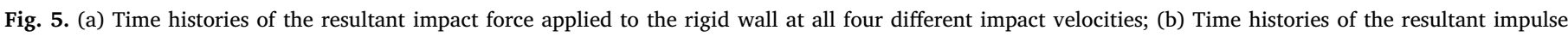
applied to the rigid wall at all four different impact velocities; (c) Normalized peak force vs. normalized square of impact velocity.

Table 4

Two velocity-dependent coefficients, the peak forces and the peak impulses ( $\alpha$ and $\gamma$ ).

\begin{tabular}{llllll}
\hline Impact velocity (m/s) & & 150 & 200 & 250 & 300 \\
$\alpha$ & & 0.6 & 0.7 & 0.83 & 0.9 \\
$\gamma$ & & 0.357 & 0.207 & 0.123 & 0.091 \\
Peak force $\left(10^{6} \mathrm{~N}\right)$ & Direct method & 76.3 & 150 & 279 & 433 \\
& Engineering model & 81.7 & 164 & 295 & 457 \\
& Error for engineering & 7.1 & 9.3 & 5.7 & 5.5 \\
& model (\%) & & & & \\
Peak impulse $\left(10^{6}\right.$ & Direct method & 7.44 & 10.75 & 14.87 & 19.07 \\
$\quad$ N.s) & Engineering model & 7.41 & 10.49 & 14.91 & 19.25 \\
& Error for engineering & -0.4 & -2.4 & 0.3 & 0.9 \\
& model (\%) & & & & \\
\hline
\end{tabular}

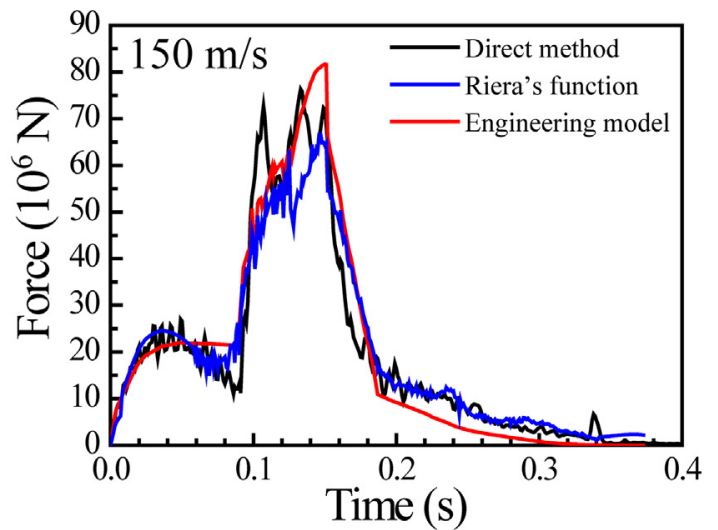

(a)

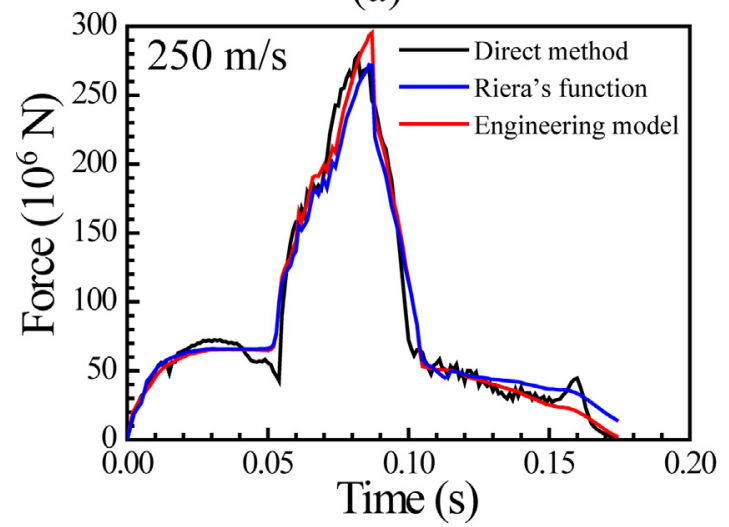

(c) strain, while the area far away from the contact surface is under elastic deformation. The debris is generated by the failure of the beam and shell elements (at the area close to the contact surface), which can be achieved using the erosion effect and the failure criteria from either Johnson-Cook model or Plastic-kinematic model. As shown in Fig. 4, the lower impact velocity can result in more left debris. The effect of impact velocity and the left debris on the coefficient $\alpha$ will be discussed later.

Fig. $5 \mathrm{a}$ and $\mathrm{b}$ show the time histories of the resultant force applied to the rigid wall and the corresponding impulse for the simulations at different impact velocities. The impulse histories were obtained by integrating the time history curves of the impact force. As indicated, the durations for the impacts of engines and wings and the durations for the impact of the entire aircraft increase with decreasing velocity, and the peak force and the maximum impulse increase with increasing impact

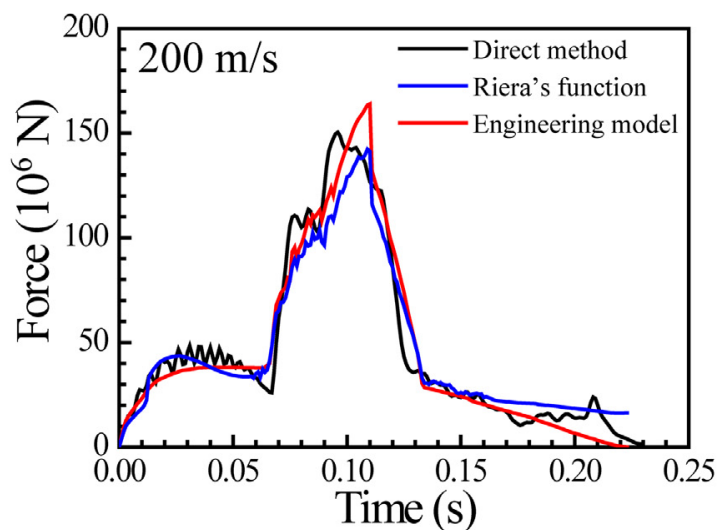

(b)

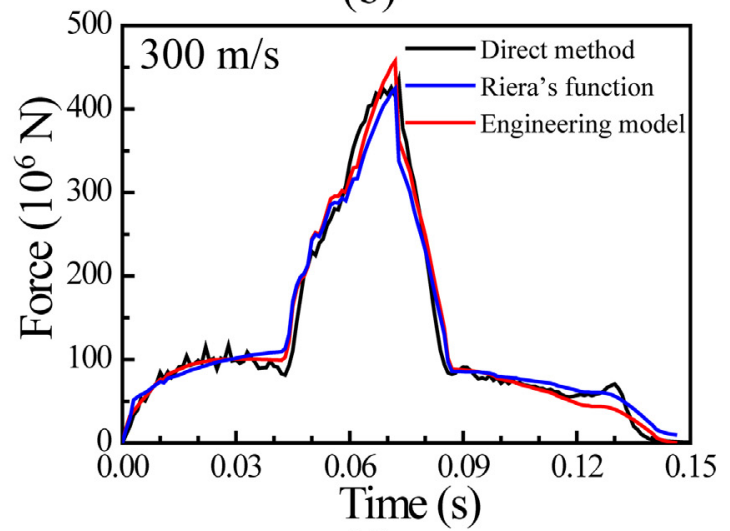

(d)

Fig. 6. Time history curves of the impact force calculated by the three methods at impact velocity of: (a) $150 \mathrm{~m} / \mathrm{s}$; (b) $200 \mathrm{~m} / \mathrm{s}$; (c) $250 \mathrm{~m} / \mathrm{s}$ (d) $300 \mathrm{~m} / \mathrm{s}$. 


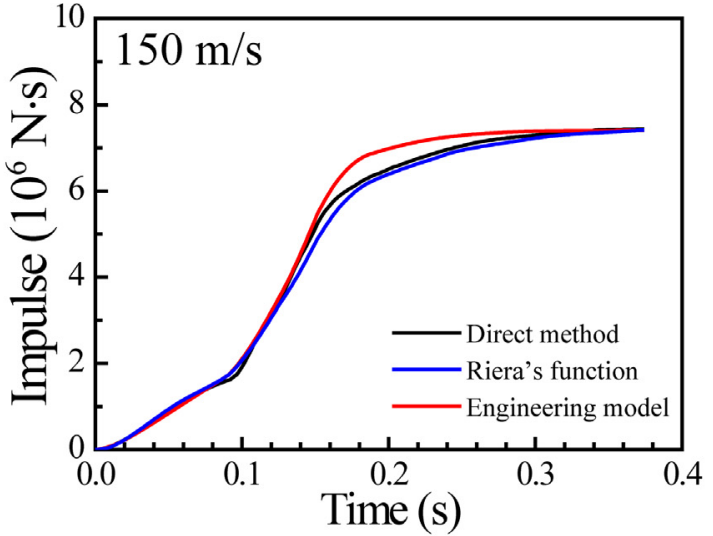

(a)

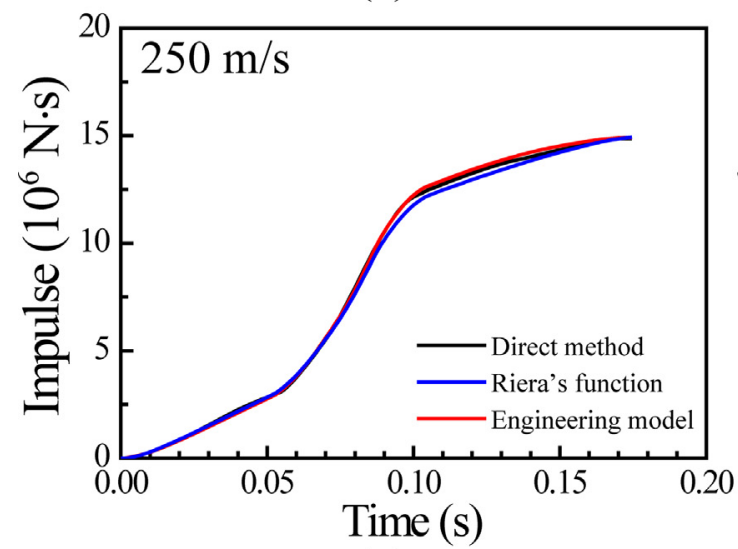

(c)

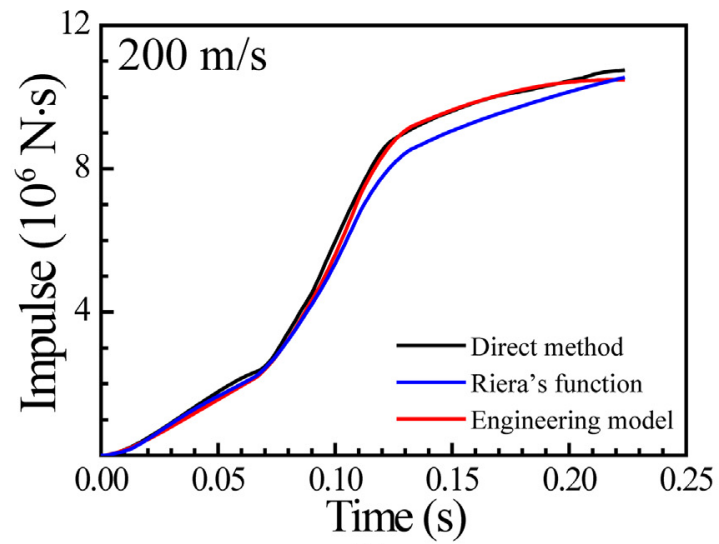

(b)

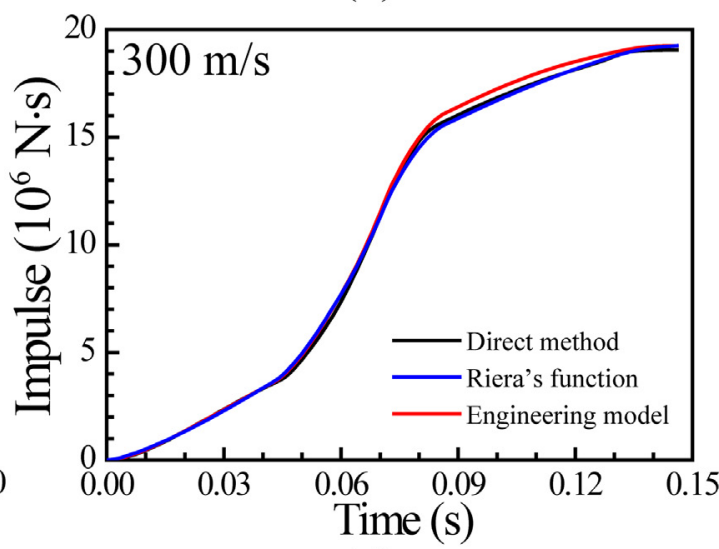

(d)

Fig. 7. Time history curves of the impulse calculated by the three methods at impact velocity of: (a) $150 \mathrm{~m} / \mathrm{s}$; (b) $200 \mathrm{~m} / \mathrm{s}$; (c) $250 \mathrm{~m} / \mathrm{s}$ (d) $300 \mathrm{~m} / \mathrm{s}$.

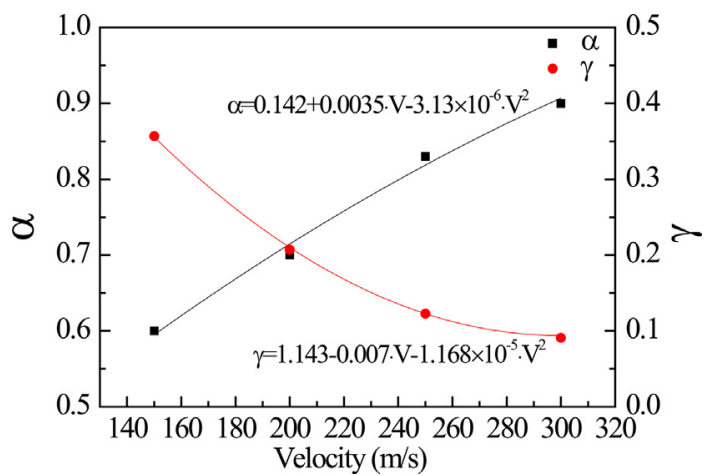

Fig. 8. Influences of the impact velocity on the two coefficients ( $\alpha$ and $\gamma$ ). Functions of two coefficients $(\alpha$ and $\gamma$ ) as the impact velocity are also obtained and displayed.

velocity. The peak force occurs earlier when the impact velocity is higher. There are two obvious inflection points in the time history curves of impulse: the first inflection point coincides with the impacts of two engines and two wings and the impulse increases sharply; while the second inflection point coincides with the end for the impacts between the two engines and two wings with the rigid wall, and the impact force decrease sharply and the increase of the impulse slows down. The normalized peak force is plotted against the normalized square of the normalized velocity in the Fig. 5c, it is indicated that the peak force has a linear relationship with the square of the impact velocity.

Besides the direct method (method I) and the indirect method (method II) for calculating the impact force history as mentioned earlier, the method by using the engineering model (Eq. (7)) can be referred to method III. However, two coefficients ( $\alpha$ and $\gamma$ ), which are both velocity-dependent, should be determined for the last two methods. Coefficient $\alpha$ can be determined by matching the time history curves of impact force and impulse for the methods (i) and (ii). Once Coefficient $\alpha$ is determined, coefficient $\gamma$ can be obtained by matching the time history curves of impact force and impulse for the methods (i) and (iii). Therefore, the two velocity-dependent coefficients ( $\alpha$ and $\gamma$ ), the peak forces and the peak impulses for the methods (i) and (iii), and the corresponding errors for the engineering model are obtained at four different impact velocities and listed in the Table 4. As indicated, the engineering model can reflect the impact force and impulse very well by choosing the appropriate values of the two coefficients, the errors for the peak force are all less than $10 \%$, and the errors for the peak impulse are all less than $2.5 \%$. The corresponding time history curves of the impact force at the four different impact velocities for the aforementioned three methods are shown in Fig. 6. The corresponding time history curves of the impulse at the four different impact velocities for the aforementioned three methods are shown in Fig. 7. As indicated, the curves of impact force and impulse for both the Riera's function and the engineering model are in good agreement with those for the direct method by choosing the appropriate values of the two velocity-dependent coefficients.

The influences of the impact velocity on the two coefficients ( $\alpha$ and $\gamma$ ) are plotted in Fig. 8, and the functions of two coefficients ( $\alpha$ and $\gamma$ ) as the impact velocity are also obtained and displayed in Fig. 8. As indicated, the reduction coefficient $\alpha$ increases with increasing impact velocity, while the coefficient $\gamma$ decreases with increasing impact velocity. As observed, the coefficient $\alpha$ is always less than a unity, similar to previous research (Lee et al., 2013). The accuracy and applicability of the Riera's function are based on the two primary assumptions (Lee 
et al., 2013): the rigid target and the axially aligned crushes/buckles. In the present study, the first assumption is always satisfied at various cases, while the second assumption might not be satisfied under the actual conditions due to the generation of debris and the effects of secondary impacts by debris. This will make the impact impulse calculated by the Riera's function deviate from the direct method. Moreover, the generation of debris causes the loss of initial kinetic energy, the lower the impact velocity, the more generated debris, and the more losses of energy and mass will be. These effects could be the reason why the reduction coefficient $\alpha$ is lower at lower impact velocities. As mentioned earlier, the static crushing force is only a small part of the total force, while this case is only true when the impact velocity is high. The coefficient $\gamma$ increases with decreasing velocity, and the static crushing force can be as large as $36 \%$ of the total impact force when the impact velocity is $150 \mathrm{~m} / \mathrm{s}$. The proposed engineering model and the obtained two velocity-dependent coefficients should provide reasonable values for the impact force with good accuracy, and should be helpful for the engineers for assessing the events of malevolent aircraft impinging on NPP. As indicated in the present study, while the peak force and total impact impulse are closely related to the total weight, the shape of the force history curve is only related to the mass distribution of the aircraft. Thus, only the results for the Boeing B737-800 aircraft are discussed in the present study, while the main conclusions can be considered to be general and can be applied to other types of large commercial aircrafts.

\section{Concluding remarks}

A commercial aircraft Boeing B737-800 is considered in order to perform numerical simulations of malevolent aircraft impinging on a rigid wall using LS-DYNA. The detailed simulations were conducted at four different impact velocities $(150,200,250,300 \mathrm{~m} / \mathrm{s}$ ) based on the well-defined FE models. The main findings are summarized as follows:

(1) There exist two distinct types of methods for obtaining the aircraft impact force: the direct method and the indirect methods. While, the static crushing force in the Riera's model is hard to be determined in practice, thus an engineering model is proposed in the present study, and the total force is only described by the dynamic force and another velocity-dependent coefficient $\gamma$.

(2) During the impact process of an aircraft crashing into an rigid wall, the generation of debris and secondary impacts make the impact impulse calculated by the Riera's function deviate from the direct method. Thus a reduction coefficient $\alpha$ should be introduced in the modified Riera's function. The coefficient $\alpha$ should be less than a unity due to the energy loss, the mass loss, the generation of debris and the effects of secondary impacts by debris.

(3) The two coefficients ( $\alpha$ and $\gamma$ ) are found to be velocity-dependent, and two formulas for the effects of impact velocity on the two coefficients are proposed. The lower impact velocity is found to result in more generated debris, thus more losses of energy and mass. Thus, the reduction coefficient $\alpha$ is found to be lower at lower impact velocities. The static crushing force is generally thought to be only a small part of the total force, which is found to be only true for high impact velocity. The coefficient $\gamma$ increases with decreasing velocity, and the static crushing force can be as large as $36 \%$ of the total impact force when the impact velocity is $150 \mathrm{~m} / \mathrm{s}$. The present findings should help the engineers to assess the events of malevolent aircraft impinging into NPP and to design the protecting shell of NPP.

\section{Declarations of interest}

None.

\section{Acknowledgments}

This work was supported by National Science and Technology Major Project of China [grant number 2013ZX06002001].

\section{Appendix A. Supplementary data}

Supplementary data to this article can be found online at https:// doi.org/10.1016/j.nucengdes.2018.11.034.

\section{References}

Abbas, H., Paul, D.K., Codbole, P.N., Nayak, G.C., 1996. Aircraft crash upon outer containment of nuclear power plant. Nucl. Eng. Des. 160, 13-50.

HyperWorks, Altair, 2010. HyperMesh. Version 11.

Andonov, A., Kostov, M., Iliev, A., 2015. Capacity assessment of concrete containment vessels subjected to aircraft impact. Nucl. Eng. Des. 295, 767-781.

Arros, J., Doumbalski, N., 2007. Analysis of aircraft impact to concrete structures. Nucl. Eng. Des. 237, 1241-1249.

Bahar, L.Y., Rice, J.S., 1978. Simplified derivation of the reaction-time history in aircraft impact on a nuclear power plant. Nucl. Eng. Des. 49, 263-268.

Boeing Website. http://www.boeing.com.

Dritter, K., Gruner, P., 1976. Calculation of the total force acting upon a rigid wall by projectiles. Nucl. Eng. Des. 37, 231-244.

Frano, R.L., Forasassi, G., 2011. Preliminary evaluation of aircraft impact on a near term nuclear power plant. Nucl. Eng. Des. 241, 5245-5250.

Hallquist, J.O., Stillman, D.W., Lin, T.-L., 2013. LS-DYNA3D user's manuals version 971. Software Technology Corporation, Livermore.

Hornyik, K., 1977. Analytic modeling of the impact of soft missiles on protective walls. In: Proceedings of 4th Conference on Structural Mechanics in Reactor Technology, San Francisco.

Hu, J.X., Lu, G., Yang, H., Yu, T.X., Xu, J., 2017. Dynamic response of internally nested hemispherical shell system to impact loading. Thin Wall. Struct. 120, 29-37.

Hu, J.X., Yin, S., Yu, T.X., Xu, J., 2018. Dynamic compressive behavior of woven flaxepoxy-laminated composites. Int. J. Impact Eng. 117, 63-74.

Iqbal, M.A., Rai, S., Sadique, M.R., Bhargava, P., 2012. Numerical simulation of aircraft crash on nuclear containment structure. Nucl. Eng. Des. 243, 321-335.

Itoh, M., Katayama, M., Rainsberger, R., 2005. Computer simulation of an F-4 Phantom crashing into a reinforced concrete wall. WIT Trans. Modell. Simul. 207-217.

Jeon, S.J., Jin, B.M., Kim, Y.J., 2012. Assessement of the fire resistance of a nuclear power plant subjected to a large commercial aircraft crash. Nucl. Eng. Des. 247, 11-22.

Jeon, S.J., Jin, B.M., 2016. Improvement of impact-resistance of a nuclear containment building using fiber reinforced concrete. Nucl. Eng. Des. 304, 139-150.

Kar, A.K., 1979. Impactive effects of tornado missiles and aircraft. J. Struct. Div. 105 (11), 2243-2260.

Kostov, M., Henkel, F.O., Andonov, A., 2014. Safety assessment of A92 reactor building for large commercial aircraft crash. Nucl. Eng. Des. 269, 262-267.

Lee, K., Han, S.E., Hong, J.W., 2013. Analysis of impact of large commercial aircraft on a prestressed containment building. Nucl. Eng. Des. 265 (6), 431-449.

Li, Q.M., Reid, S.R., Wen, H.M., Telford, A.R., 2005. Local impact effects of hard missiles on concrete targets. Int. J. Impact Eng. 32, 224-284.

Lu, X.Z., Lin, K., Cen, S., Xu, Z., Lin, L., 2015. Comparing different fidelity models for the impact analysis of large commercial aircrafts on a containment building. Eng. Fail. Anal. 57, 254-269.

Petrangeli, G., 2010. Large airplane crash on a nuclear plant: design study against excessive shaking of components. Nucl. Eng. Des. 240, 4037-4042.

Rambach, J.M., Tarallo, F., Lavarenne, S., 2005. Lavarenne airplane crash modeling: assessment of the Riera model. In: 18th International Conference on Structural Mechanics in Reactor Technology (SMiRT 18), 7-12 August, 2005, Beijing, China, pp. 2531-2538.

Riera, J.D., 1968. On the stress analysis of structures subjected to aircraft impact forces. Nucl. Eng. Des. 8, 415-426.

Riera, J.D., 1980. A critical reappraisal of nuclear power plant safety against accidental aircraft impact. Nucl. Eng. Des. 57, 193-206.

Riera, J.D., 1982. Basic concepts and load characteristics in impact problems. In: Introductory Report, RILEM-CEB-IABSE-IASS Interassociation Symposium on Concrete Structures under Impact and Impulsive Loading, 7-29 June, 1982, Berlin.

Sadique, M.R., Iqbal, M.A., Bhargava, P., 2013. Nuclear containment structure subjected to commercial and fighter aircraft crash. Nucl. Eng. Des. 260, 30-46.

Siefert, A., Henkel, F.O., 2014. Nonlinear analysis of commercial aircraft impact on a reactor building-Comparison between integral and decoupled crash simulation. Nucl. Eng. Des. 269 (4), 130-135.

Song, J.B., Li, J.L., 2016. In: Boeing 737NG Aircraft System. Aviation Industry Press, Beijing, China, pp. 5.

Sugano, T., Tsubota, H., Kasai, Y., Koshika, N., Orui, S., Von Riesemann, W.A., Bickel, D.C., Parks, M.B., 1993. Fullscale aircraft impact test for evaluation of impact force. Nucl. Eng. Des. 140, 373-385.

Tennant, D., Levine, H., Mould, J., Vaughan, D., 2014. Rapid evaluation of buildings and infrastructure to accidental and deliberate aircraft impact. Nucl. Eng. Des. 269, 142-148.

Thai, D.K., Kim, S.E., 2015. Safety assessment of a nuclear power plant building subjected to an aircraft crash. Nucl. Eng. Des. 293, 38-85. 
Wang, L.B., Yin, S., Yu, Z.X., Wang, Y.G., Yu, T.X., Zhao, J., Xie, Z.C., Li, Y.X., Xu, J., 2018. Unlocking the significant role of shell material for lithium-ion battery safety. Mater. Design 160, 601-610.

Wilt, T., Chowdhury, A., Cox, P.A., 2011. Response of Reinforced Concrete Structures to Aircraft Crash Impact Prepared for U.S. Nuclear Regulatory Commission Contract NRC-02-07-006.

Wolf, J.P., Bucher, K.M., Skrikerud, P.E., 1978. Response of equipment to aircraft impact. Nucl. Eng. Des. 47, 169-193.
Yu, G.Z., Zheng, Y.T., Feng, B., Liu, B.H., Meng, K.P., Yang, X.F., Chen, H.S., Xu, J., 2017. Computation modeling of laminated crack glass windshields subjected to headform impact. Comput. Struct. 193, 139-154.

Zhang, T., Wu, H., Fang, Q., Huang, T., 2017. Numerical simulations of nuclear power plant containment subjected to aircraft impact. Nucl. Eng. Des. 320, 207-221.

Zorn, N.F., Schueller, G.I., 1986. On the failure probability of the containment under accidental aircraft. Nucl. Eng. Des. 91, 277-286. 